\title{
AXIOMS THAT DEFINE SEMI-METRIC, MOORE, AND METRIC SPACES
}

\author{
J. R. BOYD
}

In [1] L. F. McAuley asked the following question: is it possible to partition... Moore's metrization theorem into three or more parts which begins with a condition for a topological space and which ends with a condition for a metrizable space, but with necessary and sufficient conditions somewhere between these extremes for semi-metric and Moore spaces? Axiom Z, stated below, is such a partitioning. The notation "Axiom $Z_{i}$ " denotes parts (1), (2), $\cdots$, (i) of Axiom $Z$. In $\S 1$ it is proved in Theorems 1,2 , and 3, respectively, that a necessary and sufficient condition for a topological space to be semi-metrizable, a Moore space, and metrizable is that it satisfy Axiom $Z_{2}$, Axiom $Z_{3}$, and Axiom $Z_{4}$ respectively. A counter-example is given in $\S 2$ which shows that the argument for the statement a Moore space is a semimetric topological space in Theorem 6.2 in [1] is not correct. Finally, in $\S 3$ it is shown that part (3) of Theorem 2 in [2] can be changed so that the resulting statement is equivalent to a Moore space. Definitions are given in $[1]$.

Definition. If $\left\{J_{n}\right\}$ denotes a sequence such that for each natural number $n, J_{n}$ denotes a collection of neighborhoods covering a point set $M$, then the sequence $\left\{B_{i}\right\}$, where $i$ denotes a natural number, is said to be a basic refinement of $\left\{J_{n}\right\}$ for $M$ provided that with each point $p$ in $M$ there is associated a sequence $\left\{b_{i}(p)\right\}$ such that for each $i:(1) b_{i}(p)$ is a neighborhood in $\left\{J_{n}\right\},(2) b_{i+1}(p)$ is a subset of $b_{i}(p)$, (3) $p$ is the only point common to $\left\{b_{i}(p)\right\}$, and (4) $B_{i}$ denotes the collection of all neighborhoods $b_{i}(p)$ for all points in $M$.

\section{Axiom $Z$.}

Aхгом Z. Let $T$ denote a topological space in which there exists a sequence $\left\{J_{n}\right\}$ such that:

(1) for each natural number $n, J_{n}$ denotes a collection of neighborhoods in $T$ covering $T$,

(2) there exists a basic refinement $\left\{B_{n}\right\}$ of $\left\{J_{n}\right\}$ for $T$ such that if $M$ denotes a point set and $p$ denotes a point, then either (a) every neighborhood containing $p$ contains a point in $(M-p)$ or $(\mathrm{b})$ there exists an $n$ such that if $x$ denotes $a$ point in $(M-p), b_{n}(p)$ does not contain $x$ and $b_{n}(x)$ does not contain $p$,

(3) if $R$ denotes a neighborhood containing $p$ and $x$ is in $R$, then there

Presented to the Society, December 6, 1960; received by the editors January 13, 1961 and, in revised form, April 4, 1961. 
exists an $n$ such that if $g$ denotes a neighborhood in $J_{n}$ that contains $p$, then $\bar{g}$ is a subset of $R$ not containing $x$ unless $x$ is $p$, and

(4) if $R$ contains $p$ and $x$ is different from $p$, then there exists an $n$ such that if each of $h$ and $k$ denotes $a$ neighborhood in $J_{n}, k$ contains $p$, and $h$ and $k$ have a common part, then $h$ is a subset of $R$ not containing $x$.

Parts (1) and (4) are Moore's metrization theorem and parts (1) and (3) are parts (1) and (3) of Moore's Axiom $1_{3}$.

Theorem 1. A necessary and sufficient condition for a topological space $T$ to be semi-metrizable is that $T$ satisfy Axiom $Z_{2}$.

Proof of NECEssity. It follows from the definition of a semi-metric topological space that for each point $p$ in $T$, there exists a sequence $\left\{b_{n}(p)\right\}$ such that for each $n,(1) b_{n}(p)$ denotes a neighborhood containing $p,(2) b_{n}(p)$ is a subset of the $1 / n$-neighborhood of $p$, and (3) $b_{n+1}(p)$ is a subset of $b_{n}(p)$. Now, for each $n$, let $B_{n}$ denote the collection of all the neighborhoods $b_{n}(p)$ for the various points in $T$. Finally, for natural numbers $i$ and $m$ let $J_{m}$ denote the collection of all the elements in $B_{i}$ for all $i \geqq m$. It is not difficult to verify that Axiom $Z_{2}$ is satisfied by the sequences $\left\{J_{n}\right\}$ and $\left\{B_{n}\right\}$.

Proof of sufficiency. For each pair of points $p$ and $x$ in $T$, let $n$ denote the smallest $i$ such that $b_{i}(x)$ does not contain $p$ and $b_{i}(p)$ does not contain $x$ where $b_{i}(x)$ and $b_{i}(p)$ belong to $B_{i}$. Now let $d(p, x)=d(x, p)=1 / n$. Define $d(p, p)=0$. It follows that $d$ is a semimetric for $T$ and that the sufficiency of the condition is established.

Theorem 2. A necessary and sufficient condition for a topological space $T$ to be a Moore space is that $T$ satisfy Axiom $Z_{3}$.

Proof of Necessity. Let $\left\{G_{n}\right\}$ denote the sequence of collections of neighborhoods given in Moore's Axiom 1 $1_{3}$. Since a Moore space is a semi-metric space [1], define the sequences $\left\{B_{n}\right\}$ and $\left\{J_{n}\right\}$ in a manner analogous to that in the proof of the necessity of Theorem 1 with the additional restriction that each member of $J_{n}$ be a subset of an element of $G_{n}$, i.e., $J_{n}$ refines $G_{n}$ for each $n$. It follows that the sequences $\left\{J_{n}\right\}$ and $\left\{B_{n}\right\}$ satisfy Axiom $Z_{3}$.

Proof of Sufficiency. Given that $T$ satisfies Axiom $Z_{3}$, let $G_{n}$ denote the collection of all elements $B_{i}$ for all $i \geqq n$. It follows that the sequence $\left\{G_{n}\right\}$ satisfies Moore's Axiom $1_{3}$.

TheOREM 3. A necessary and sufficient condition for a topological space $T$ to be metrizable is that $T$ satisfy Axiom $\mathrm{Z}_{\mathbf{4}}$.

2. A correction. The following counter-example shows that the argument for the statement a Moore space is a semi-metric topological space in $[1$, Theorem 6.2] is not correct. 
Let the points of space be the points of the plane on or above the $x$-axis. Neighborhoods are of two types-interiors of circles above the $x$-axis and interiors of circles tangent to the $x$-axis from above together with the point of tangency. Let $G_{i}$ denote the collection of all these neighborhoods whose diameters are less than $1 / i$.

McAuley defined the distance between two points $p$ and $q$ as follows: denote by $n$ the least positive integer such that if $g(p)$ and $g(q)$ denote two neighborhoods in $G_{n}$ containing the points $p$ and $q$ respectively, then $g(p) \cdot g(q)=0$. Define $d(p, q)=1 / n$. By this definition, a point on the $x$-axis is a distance limit point of the $x$-axis but is not a limit point of the $x$-axis.

It is not difficult to show that the following definition of distance is sufficient to show that a Moore space is a semi-metric topological space. Let $n$ denote the least natural number $i$ such that if $g(p)$ and $g(q)$ are two neighborhoods in $G_{i}$ containing $p$ and $q$ respectively, then $g(p)$ does not contain $q$ and $g(q)$ does not contain $p$. Define $d(p, q)=1 / n$.

3. Axiom A. R. L. Moore's Axiom $1_{3}$ is stated in terms of a sequence of collections of regions covering space. The following axiom is stated in terms of a point and a sequence of regions containing the point. The axiom is the same as Theorem 2 in [2] except for part (3).

Axiom A. If $p$ denotes a point, there exists a sequence $\left\{R_{n}(p)\right\}$ where for each natural number $n, R_{n}(p)$ denotes a region containing $p$ such that:

(1) $p$ is the only point common to $\left\{R_{n}(p)\right\}$,

(2) for each $n, R_{n+1}(p)$ is a subset of $R_{n}(p)$,

(3) if $R$ denotes a region containing $p$, then there exists an $n$ such that if $z$ denotes any point and $R_{n}(z)$ contains $p, \bar{R}_{n}(z)$ is a subset of $R$.

Theorem 4. In the presence of Moore's Axiom 0, Axiom $1_{3}$ is equivalent to Axiom A.

Proof. That Axiom $1_{3}$ implies Axiom $\mathrm{A}$ is established by obtaining the sequence $\left\{R_{n}(p)\right\}$ as in the proof of Theorem 2 in [2] and showing that part (3) of Axiom A follows from part (3) of Axiom $1_{3}$. To show that Axiom A implies Axiom $1_{3}$, let $G_{n}$, for each $n$, denote the collection of all regions $R_{i}(p)$ for all natural numbers $i \geqq n$ for all points $p$. It is easy to verify that the sequence $\left\{G_{n}\right\}$ satisfies Axiom $1_{3}$.

\section{REFERENCES}

1. L. F. McAuley, A relation between perfect separability, completeness, and normality in semi-metric spaces, Pacific J. Math. 6 (1956), 315-326.

2. R. L. Moore, Foundations of point set theory, Amer. Math. Soc. Colloq. Publ. Vol. 13, Amer. Math. Soc., New York, 1932.

Arlington State College 